EXTENDED REPORT

\title{
The relation of volume with outcome in phacoemulsification surgery
}

\author{
M Habib, K Mandal, C V Bunce, S G Fraser
}

Br J Ophthalmol 2004;88:643-646. doi: 10.1136/bjo.2003.028159

See end of article for authors' affiliations ......................

Correspondence to: Mr S G Fraser, Sunderland Eye Infirmary, Queen Alexandra Road, Sunderland SR2 9HP, UK; sfraser100@totalise.co.uk

Accepted for publication 22 September 2003

\begin{abstract}
Background/aims: High case volume has been associated with better health outcomes for a variety of procedures and conditions including coronary angioplasty, carotid endarterectomy, colorectal surgery, and various types of cancer surgery. The association of volume and outcome has important implications for patient safety and healthcare delivery planning. The relation between surgical volume and outcome has not, as far as is known, been looked at for phacoemulsification alone.

Methods: All cataract surgery performed from 1996 to 2001 by six consultant surgeons was reviewed. Using theatre logbooks and cross checking with the hospital database, the total number of phacoemulsification procedures performed per surgeon per year was calculated. The total number of operations in which it was judged that significant intraoperative complications occurred was also counted. Results: When the data were pooled for all the surgeons there was evidence that complication rate decreased over time (Spearman's rho $=-0.319, p=0.058$ ). If the data were pooled from all the years and all the surgeons then there was strong evidence of a decrease in complication rate with an increase in the number of cases (Spearman's rho $=-0.63, \mathrm{p}<0.01$ ).

Conclusions: This study is the first to describe a possible relation between volume of surgery and the outcome (as measured by complication rates) for phacoemulsification. There are however some caveats in that the issue of case mix was not addressed and that the results are from a single unit and may not necessarily be generalisable
\end{abstract}

$\mathrm{H}$ igh case volume has been associated with better outcomes for a variety of procedures and conditions including coronary angioplasty, carotid endarterectomy, colorectal surgery, and various types of cancer surgery. ${ }^{1}$ The association of volume and outcome has important implications for patient safety and for healthcare delivery and planning.

There is very little ophthalmic literature that addresses the relation between volume and outcome. Cataract surgery using phacoemulsification is one of the commonest surgical procedures $^{2}$ in Europe and North America yet, as far as we know, there have not been any studies that looked solely at surgical volume as a determinant of outcome (as measured by complication rate). This study was designed as a first step in analysing the presence or absence of any such relation.

\section{MATERIALS AND METHODS}

The study was conducted wholly at Sunderland Eye Infirmary. This is a single specialty ophthalmic hospital geographically separated from the main general hospital. It has its own dedicated ophthalmic operating theatres and the operating theatre staff are not rotated to other departments or sites. Medical records are contained on site which also has the benefit of dedicated audit staff and a specialty specific patient database.

Data were collected retrospectively from the first operation in January 1996 to the final operation in December 2001that is, six complete calendar years. Entry criteria for the study were:

- Cataract surgery performed with the intention that the nucleus would be removed by phacoemulsification.

- Surgery performed by a consultant ophthalmologist.

A total of 16975 cases were identified that conformed to these criteria over the 6 years.
The data were collected using hand searching of theatre logbooks for this period of time and cross checking with the hospital database. The data collected and checked from these sources were:

(1) Date of operation

(2) Name of surgeon performing operation

(3) Significant complications that were noted to have occurred during the operation. These consisted of:

(a) Posterior capsule rupture with or without anterior vitrectomy

(b) Loss of nuclear fragments into the vitreous

(c) Sulcus placed posterior chamber lens

(d) Anterior chamber lens implantation.

If more than one of these complications occurred it was noted only as one event.

The data were collated by an experienced ophthalmic trainee and double checked by a consultant ophthalmologist (who was not one of the surgeons operating on the patients).

\section{RESULTS}

Six consultant ophthalmologists who removed cataracts with phacoemulsification and who operated throughout the 6 years of the study were included. Table 1 shows the number of phacoemulsifications (phacos) performed and the total number of complications for each surgeon for each year of the study. Table 2 expresses these data as complication percentages per surgeon per year.

When the data were pooled for all the surgeons there was evidence that complication rate decreased over time (Spearman's rho $=-0.319, \mathrm{p}=0.058$ ). If the data was then pooled from all the years and all the surgeons then there was strong evidence of a decrease in complication rate with an 
Table 1 Number of phacoemulsifications (phacos) performed and the total number of complications for each surgeon for each year of the study

\begin{tabular}{|c|c|c|c|c|c|c|c|c|c|c|c|c|}
\hline \multirow{2}{*}{$\begin{array}{l}\text { Year } \\
\text { No cases and } \\
\text { complications }\end{array}$} & \multicolumn{2}{|l|}{1996} & \multicolumn{2}{|l|}{1997} & \multicolumn{2}{|l|}{1998} & \multicolumn{2}{|l|}{1999} & \multicolumn{2}{|l|}{2000} & \multicolumn{2}{|l|}{2001} \\
\hline & Cases & Comp & Cases & Comp & Cases & Comp & Cases & Comp & Cases & Comp & Cases & Comp \\
\hline \multicolumn{13}{|l|}{ Surgeon } \\
\hline 1 & 617 & 10 & 772 & 15 & 896 & 13 & 1106 & 8 & 1161 & 9 & 1000 & 14 \\
\hline 2 & 408 & 12 & 413 & 7 & 484 & 14 & 658 & 5 & 829 & 9 & 791 & 9 \\
\hline 3 & 530 & 7 & 623 & 6 & 769 & 6 & 947 & 6 & 792 & 3 & 569 & 2 \\
\hline 4 & 300 & 11 & 341 & 9 & 364 & 11 & 361 & 11 & 353 & 16 & 307 & 5 \\
\hline 5 & 119 & 6 & 150 & 6 & 184 & 3 & 123 & 3 & 66 & 2 & 50 & 1 \\
\hline 6 & 113 & 4 & 220 & 2 & 211 & 2 & 243 & 5 & 55 & 2 & 48 & 1 \\
\hline Total & 2087 & 50 & 2519 & 45 & 2908 & 49 & 3438 & 38 & 3256 & 41 & 2765 & 32 \\
\hline
\end{tabular}

increase in the number of cases (Spearman's rho $=-0.63$, $\mathrm{p}<0.01)$. Figure 1 compares the number of cases performed in any year and by any surgeon with the percentage complications associated with that number.

We attempted to make comparisons between surgeons by dividing the surgeons into "high" and "low" volume. High volume surgeons were designated as those who performed more than 400 cases per year while low volume was taken as fewer than 400 cases per year (this figure was arrived at by looking at the figures and by comparison with the published literature). This meant that three surgeons were considered high volume and three low volume. In the majority of years there was little overlap between the groups with the difference between those considered low and those considered high usually at least 200 cases. Table 3 compares the average complication rate of high volume surgeons with low volume throughout the study period. This is illustrated in figure 2 .

The individual data for different surgeons did not always show a statistically different decrease in complication rate either over time or with increased volume.

\section{DISCUSSION}

In modern healthcare systems there will always be financial, patient safety, and legal pressures to assess outcomes. Accurate and valid health outcomes are not easy to evaluate and may, sometimes, only be obtained with unjustifiable expenditure. Data on volume appear a relatively cheap and simple measure of outcomes and for this reason, measuring and understanding the relation between volume and outcome in the delivery of health services has been the focus of much research since the $1980 \mathrm{~s}^{2}{ }^{3}$ There are a number of comprehensive reviews in the published literature. ${ }^{13}$

Good evidence exists that certain procedures (although not all) show a direct relation between increased volume and improved quality of outcomes-that is, bigger numbers means better results (mortality and/or morbidity). ${ }^{4}$ It has been suggested that there are two possible reasons for this relation ${ }^{3}$ :

(1) Physicians (and hospitals) develop more effective skills if they treat more patients (practice makes perfect), or

(2) Physicians (and hospitals) achieving better outcomes receive more referrals and thus accrue larger volumes (selective referral).

Volume and outcome studies look at either hospital or clinician volume-although a few studies have looked at both. Hospital volume studies are those that compare data for one or more procedures between low and high volume hospitals. Physician or clinician volume studies (such as this study) compare data between high and low volume doctors. The main areas that have been studied are cardiac procedures and cancer treatment but other areas, both medical and surgical, have been explored and there are some examples of these listed below.

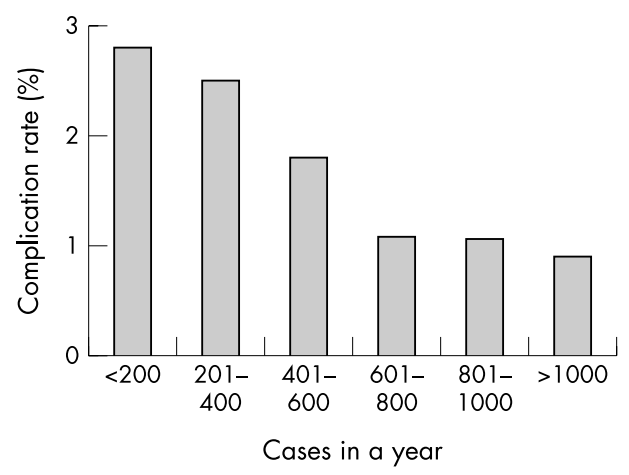

Figure 1 Complication rate (\%) versus number of cases performed in the year.

Table 2 Number of phacos performed and percentage that were complicated for each surgeon and for each year of the study

\begin{tabular}{|c|c|c|c|c|c|c|c|c|c|c|c|c|}
\hline \multirow{2}{*}{$\begin{array}{l}\text { Year } \\
\begin{array}{l}\text { No cases and \% } \\
\text { complications }\end{array}\end{array}$} & \multicolumn{2}{|l|}{1996} & \multicolumn{2}{|l|}{1997} & \multicolumn{2}{|l|}{1998} & \multicolumn{2}{|l|}{1999} & \multicolumn{2}{|l|}{2000} & \multicolumn{2}{|l|}{2001} \\
\hline & Cases & $\%$ & Cases & $\%$ & Cases & $\%$ & Cases & $\%$ & Cases & $\%$ & Cases & $\%$ \\
\hline \multicolumn{13}{|l|}{ Surgeon } \\
\hline 1 & 617 & 1.6 & 772 & 1.9 & 896 & 1.5 & 1106 & 0.7 & 1161 & 0.8 & 1200 & 1.2 \\
\hline 2 & 408 & 2.9 & 413 & 1.7 & 484 & 2.9 & 658 & 0.8 & 829 & 1.1 & 791 & 1.1 \\
\hline 3 & 530 & 1.3 & 623 & 1 & 769 & 0.8 & 947 & 0.6 & 792 & 0.4 & 569 & 0.4 \\
\hline 4 & 300 & 3.7 & 341 & 2.6 & 364 & 3 & 361 & 3.1 & 353 & 4.5 & 307 & 1.6 \\
\hline 5 & 119 & 5 & 150 & 4 & 184 & 1.6 & 123 & 2.4 & 66 & 3 & 50 & 2 \\
\hline 6 & 113 & 3.5 & 220 & 1 & 201 & 1 & 243 & 2.1 & 55 & 1.9 & 48 & 2.1 \\
\hline
\end{tabular}


Table 3 Comparison of average complication rate of high volume surgeons ( $>400$ cases per year) with low volume $(<400)$ throughout the study period

\begin{tabular}{lllllll}
\hline \% complication rates & 1996 & 1997 & 1998 & 1999 & 2000 & 2001 \\
\hline High vol surgeon $(>400$ cases per year) & 1.9 & 1.5 & 1.7 & 0.7 & 0.8 & 0.9 \\
Low volume surgeon $(<400$ cases per year) & 4.1 & 2.5 & 1.9 & 2.5 & 3.1 & 1.9 \\
\hline
\end{tabular}

- Coronary artery bypass surgery ${ }^{5}$-reduced risk of in-hospital mortality in hospitals carrying out more than 200 procedures/year.

- Paediatric cardiac surgery ${ }^{6}$ - UK hospitals (excluding Bristol) carrying out 120 open heart operations on children aged under 1 year would be expected to have a mortality $25 \%$ lower than that in a hospital carrying out 40 operations.

- Knee and hip replacement ${ }^{7}$-higher hospital volume associated with lower risk of complications.

- Breast cancer $-15 \%$ reduction in mortality with surgeons treating $>29$ new cases/year.

- Intestinal operations (excluding cancer) ${ }^{9}$ - surgeons with annual volume greater than eight associated with a lower mortality.

Further details on these procedures and others can be found in work by Halm et al, ${ }^{3}$ Birkmeyer et $a l,{ }^{4}$ and a review from the NHS Centre for Reviews and Dissemination. ${ }^{1}$

As far as ophthalmology is concerned, there has been only one study that has looked into the possibility of a relation between variations in cataract clinical practice and outcomes. ${ }^{10}$ Schein et al conducted a prospective cohort study of 772 US patients undergoing first eye cataract extraction. Operating surgeons from a number of hospitals were involved. The volume of cataract surgery for each surgeon was calculated and stratified as moderate (51-200), high (201-399) or very high (>400 cases per year). Surgery was performed by either standard extracapsular cataract surgery (ECCE) or phacoemulsification.

Data were collected with regard to adverse events and Snellen visual acuity at 4 months. The results suggested that there was no statistically significant association between volume and final visual acuity. It was however noted that the high and very high volume surgeons had a higher reported occurrence of posterior capsule opacification at 4 months.

The advantage of the Schein study was that it was prospective (unlike our study), which usually means that data collection is more likely to be complete. However, the total number of patients was small compared to our study and there was the added complication that two different cataract extraction techniques were being used by the

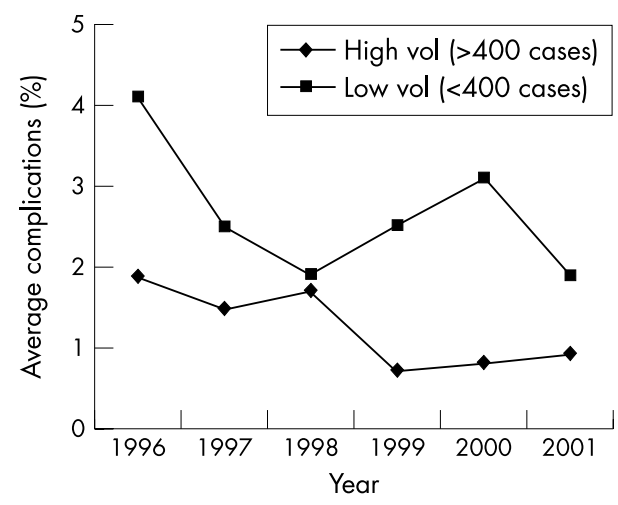

Figure 2 Comparison of average complications for high volume surgeons versus low volume surgeons. surgeons. Both our study and Schein's study use rather crude measurements of complications of cataract surgery and it is likely that more subtle complications occur but are not recorded. There is, however, no reason to think that these subtler complications would be differentially spread between the lower and higher volume groups in either study. The advantage of a single hospital, as in our study, is that it reduces some of the variations in practice/environment that occur with multiple sites. Data collection was relatively simple and only one computer system was needed with the vast majority of patient notes being readily available. Using one site does however raise the question regarding the national or international generalisability of the findings. It is thus important to repeat this study in other units using a standard methodology so that accurate comparisons can be made.

The results of our study show that complication rates for low and high volume surgeons decrease over time. This is expected and is the result of increasing familiarity with the technique and improvements in equipment. The results also indicate a trend towards fewer complications the more phacoemulsification procedures a surgeon performs. This result is not statistically significant for the individual surgeon but comes from pooling the data. This trend is shown in table 3 and illustrated in figure 2 and indicated that when the average complication rate of low and high volume surgeons is compared, it can be seen that low volume surgeons always had a higher average complication rate than the high volume ones. It is important to note that the absolute risk between high and low volume surgeons would be small and that all surgeons had complications rates within published guidelines.

What is more interesting is the lowering of the complication rate as the volume performed in any particular year increased (that is, the surgeons had lower complication rates in years when they performed more cases). This was statistically significant $(\mathrm{p}<0.01)$ and is pictorially represented by the bar chart in figure 1 (note: this is for all surgeons and for any year). It is important to realise that this lowering was independent of the year on year improvement and is at least suggestive of a volume/outcome relation.

Our study did not address quality issues such as visual outcome and patient satisfaction (using complication rate as a surrogate for these) but there have been a number of studies which have shown that intraoperative complications tend to be associated with poorer final visual acuity. ${ }^{11}$

It is impossible to discuss the relation of volume and outcome without addressing the issue of case mix. Unmeasured differences in patient populations between doctors or hospital with different volumes will result in confounding of the results. ${ }^{32}$ Some surgeons may be doing a larger proportion of the more difficult cases than other surgeons. This means they are likely to be doing operations that take more time (so they have a reduced patient volume) and are more likely to have complications (because they are operating on more difficult cases). It is obvious that comparisons of surgeons who do more straightforward operations with those who do more complicated operations will be misleading. Not surprisingly, it has been shown that 
the more volume/outcome data are adjusted for case mix the weaker is the relation between increased volume and improved outcome. ${ }^{12}$

As far as cataract surgery is concerned, it may be reasonable to assume that case mix is fairly even spread between most surgeons. In our study we did not look at case mix, but experience in the unit suggests that the three high volume surgeons were doing at least as many potentially complicated case as the low volume surgeons. In fact, it may be that the high volume surgeons were doing more of the complicated cases because they were seen as more able to deal with them (selective referral). This would, contrary to most case mix confounding, mean that their overall complication rate, if risk adjusted, would make the results of high volume surgeons look even better. Because of the importance of the effect of case mix, we are addressing it in a follow up study.

If the findings of our study are correct what is the mechanism of lower complications with increased volume of cases? One possibility is the "practice makes perfect" hypothesis in that because the high volume surgeons were doing more case they were simply getting better at doing the operations. A further possibility, that is not mentioned in the literature, is that "better" surgeons (that is, those with fewer complications) are more able (or willing) to do high case volumes. This would of course only hold if the lower volume surgeons were not doing more of the difficult cases. The "selective referral" hypothesis seems the least likely to explain the findings, as in a publicly funded healthcare system such as the NHS, it would be very unusual for a surgeon to have a limit to his/her cataract surgery workload because of lack of referrals.

In conclusion, this study is the first to describe a possible relation between volume of surgery and the outcome (as measured by complication rates) for phacoemulsification. There are however some caveats to add in that the issue of case mix was not addressed and that the results are from a single unit and may not necessarily be generalisable. If other units do have similar results, there are important implications for training as well as for public health policy, health service planning, and, not least, with regard to patient safety.

\section{Authors' affiliations}

M Habib, K Mandal, S G Fraser, Sunderland Eye Infirmary, Sunderland, UK

C V Bunce, Moorfields Eye Hospital, London, UK

\section{REFERENCES}

1 NHS Centre for Reviews and Dissemination. Hospital volume and healthcare outcomes, costs and patient access. Effective Healthcare Bulletin (2)8. York: University of York, 1996.

2 Spalton D, Koch D. The constant evolution of cataract surgery. BMJ 2000;321:1304

3 Halm EA, Lee C, Chassin MR. Is volume related to outcome in health care? A systematic review and methodologic critique of the literature. Ann Intern Med 2002;137:51 1-21.

4 Birkmeyer JD, Siewers AE, Finlayson EVA, et al. Hospital volume and surgical mortality in the United States. N Engl J Med 2002;346:1 128-37.

5 Sowden AJ, Deeks JJ, Sheldon TA. Volume and outcome in coronary artery bypass graft surgery: true association or artefact? BMJ 1995;311:151-5.

6 Spiegelhalter DJ. Mortality and volume of cases in paediatric surgery: retrospective study based on routinely collected data. BMJ 2001;323:1-5.

7 Taylor HD, Dennis DA, Crane HS. Relationship between mortality rates and hospital volume for Medicare patients undergoing major orthopaedic surgery of the hip, knee, spine and femur. J Arthroplasty 1997;12:235-42.

8 Sansbury R, Haward B, Rider I, et al. Influence of clinician workload and patterns of treatment on survival from breast cancer. Lancet 1995;345: 1265-70.

9 Hannan El, O'Donnel JF, Kilburn H, et al. Investigation of the relationship between volume and surgical procedures performed in New York state hospitals. JAMA 1989;262:503-10.

10 Schein OD, Steinberg EP, Javitt JC, et al. Variation in cataract surgery practice and clinical outcomes. Ophthalmology 1994;101:1142-52.

11 lonides A, Minassian D, Tuft S. Visual outcome following posterior capsule rupture during cataract surgery. $\mathrm{Br} J$ Ophthalmol 2001;85:222-4.

12 Soljak M. Volume of procedures and outcome of treatment. BMJ 2002;325:787-8. 